\title{
A Distinctive Case of Acute Alveolar Hyaline Membrane Formation In Microscopic Polyangiitis.
}

\author{
Qaisar Huzaif*, Zheng Min, Tang Xiaoyin, Akula Monika and Kyzyshyn Halyna
}

Jersey Shore University Medical Center, NJ, USA.

Received: 06 April, 2018; Accepted: 12 May, 2018; Published: 28 May, 2018

*Corresponding author: Qaisar Huzaif, Jersey Shore University Medical Center, NJ, USA. E-mail: huzaif.qaisar@hackensackmeridian.org

\begin{abstract}
Microscopic Poly Angiitis (MPA) is a systemic necrotizing vasculitis that affects small vessels and is without granulomatous formation. The kidneys and lungs are the most frequently affected organs. The pulmonary manifestations of microscopic polyangiitis are diverse and range from antecedent interstitial fibrosis to alveolar hemorrhage secondary to diffuse alveolar damage and pulmonary capillaritis. Awareness of this condition and a prompt diagnosis is warranted as this is severe and life threatening condition. The need to distinguish true primary systemic vasculitis from its multiple potential mimickers is one of the most challenging diagnostic puzzles in clinical medicine. We are presenting a case with finding of acute alveolar hyaline membrane formation on open lung biopsy in a patient fulfilling criteria of MPA. Once diagnosed, this life threatening condition has to be treated with systemic steroids, Rituximab or Cyclophosphamide induction therapy, as well as plasmapheresis in some cases.
\end{abstract}

Keywords: Microscopic polyangiitis; Alveolar hyaline membrane formation;

\section{Introduction}

We describe a case of microscopic polyangiitis with alveolar hyaline membrane formation as lung biopsy finding. Among the most challenging aspects of evaluating and caring for patients with systemic vasculitis is the need to distinguish rigorously between vasculitis and a host of conditions that can mimic vasculitis closely [1]. Understanding biopsy findings of MPA is of utmost importance because of ambiguous and variable nature of this disease [2,3]. Clinical presentations may vary from constitutional symptoms to classic pulmonary-renal syndrome and other organs involvement $[4,5]$. A positive Anti-Neutrophil Cytoplasmic Antibodies (ANCA) test strongly suggests the diagnosis of vasculitis, but both diagnostically false-positive and false-negative results can be seen [6, 7]. Histological examination of tissue obtained by biopsy of an involved organ (generally, skin, kidney, nerve or lung) remains the most definitive way to establish a diagnosis and is very so often required [8]. The presence of vasculitis in vessels smaller than arteries, such as arterioles, venules and capillaries is usually present [9].The paucity of immunoglobulin is demonstrable using immunofluorescence microscopy (ie, pauci-immune injury) $[10,11]$.

Patient in this case presented with multi-lobar pneumonia symptoms with pleuritic chest pain and pleural effusion associated with constitutional symptoms that included arthralgia, malaise, weight loss and fever. Unresolving symptoms on optimal treatment of pneumonia and nondiagnostic flexible bronchoscopy lead to suspicion of other pathology. Final diagnosis of MPA was made when high titers of perinuclear Anti-Neutrophil Cytoplasmic Antibodies (p-ANCA) and Myeloperoxidase (Mpo) came back positive and open lung biopsy revealed prominent alveolar hyaline membrane formation, foci of leukocytoclastic angiitis, focal organizing pneumonia, and interstitial fibrosis. This opened a new horizon to further study and include alveolar hyaline membrane formation as part of biopsy finding in MPA rather than only considering this finding in Acute Respiratory Distress Syndrome (ARDS) patients [12,13].

\section{Case presentation}

A 50-year-old Caucasian woman with a remote history of meconium induced pneumonia in infancy leaving her with permanent scarring of the right middle lobe was diagnosed with right sided community acquired pneumonia as outpatient and completed 10-day course of levofloxacin without improvement. She presented to the hospital on $11 / 25 / 17$ with worsening dry cough, right sided pleuritic chest pain and fevers up to $38.3 \mathrm{C}$. She also reported generalized body aches, bilateral knee pain and mild weight loss. Physical examination revealed heart rate $120 / \mathrm{min}$, oral temperature $37.3 \mathrm{C}$, respiratory rate $20 / \mathrm{min}, 02$ saturation $94 \%$ on room air, blood pressure $129 / 87 \mathrm{mmHg}$. On auscultation, clear breath sounds on the left and decreased breath sounds on the right throughout. No wheezes, rhonchi or rales appreciated. Heart, abdominal, musculoskeletal and neurologic exam was normal. No skin rash appreciated. Laboratory data on presentation revealed elevated White Bloodcell Count (WBC) $13.1 \mathrm{k} / \mathrm{ul}$ (85\% neutrophils, $12.1 \%$ lymphocytes, monocytes $4.2 \%$ and eosinophils $2.2 \%$ ), elevated platelet count $632 \mathrm{k} /$ ul (140-450 k/ul), Hemoglobin (Hb) 11.9 (12.0-16.0 gm/dl) with baseline $\mathrm{Hb}$ of $13.6 \mathrm{gm} / \mathrm{dl}$ two weeks prior to admission and Erythrocyte Sedimentation Rate (ESR) of 100 (0-30 mm/ hr), blood cultures were negative. Legionella urine antigen, streptococcus urine antigen, mycoplasma IgM, nasal Influenza A and B, HIV, Quantiferon TB gold tests were negative. On the day of admission Computed Tomography (CT scan) of the chest revealed large right sided consolidation with associated right- 

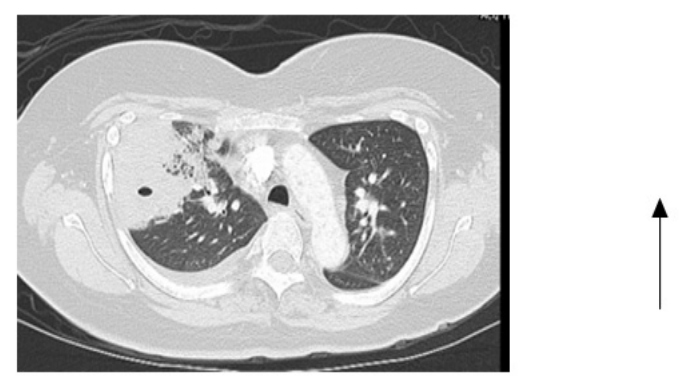

Figure 1: CT chest 11/26/17 Large right sided consolidation and left sided pulmonary nodules

sided pleural effusion and small left lung nodules (Figure 1).

Right sided thoracentesis was performed and 1 liter of serosanguineous fluid was drained. Pleural fluid analysis showed exudative picture (protein $4 \mathrm{gm} / \mathrm{dl}$, LDH $237 \mathrm{iU} / \mathrm{L}$ and WBC 9476, with serum LDH 228 (91-200 iU/L) and serum protein of $4.8(6-8 \mathrm{~g} / \mathrm{dL})$, negative cultures and no malignant cells. She was treated with broad-spectrum antibiotics for suspected pneumonia possibly secondary to resistant organisms. Two days later she underwent flexible bronchoscopy with BronchoAlveolar Lavage (BAL) and trans bronchial biopsy. BAL fluid analysis was negative for signs of alveolar hemorrhage, malignant cells, and infection including Acid-Fast Bacilli (AFB) and fungal culture. Results of the right upper and middle lobe lung biopsy through the trans-bronchial approach showed acute on chronic non-necrotizing granulomatous inflammation. Further workup that included coccidioides antibody, histoplasma urine antigen and serum cryptococcus antigen were negative. On the second week of hospitalization, repeat computed tomography of the chest (Figure 2) was done due to lack of clinical response to treatment and it showed worsening large areas of parenchymal consolidation in the right lung, new left upper lobe and new right lower lobe airspace infiltrates. Patient's condition deteriorated with worsening shortness of breath, increased work of breathing with respiratory rate of $24 / \mathrm{min}$ and 02 saturation $94 \%$ despite BIPAP.
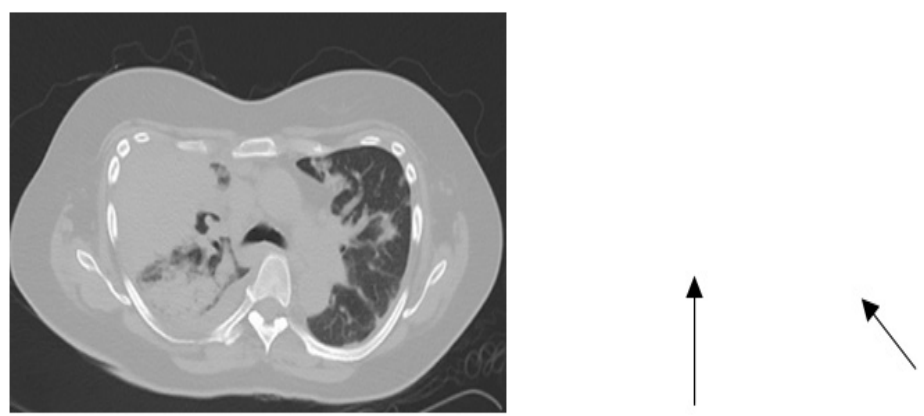

Figure 2: CT chest 12/4/17 Worsening large areas of parenchymal consolidation in the right lung and patchy infiltrate in the left upper lobe.

She was intubated on fourteenth day of hospitalization for increased work of breathing and worsening hypoxia with respiratory rate of $22 /$ minute. Laboratory data at the time of intubation included a hemoglobin drop from $9.6 \mathrm{~g} / \mathrm{dL}$ to $6.9 \mathrm{~g} /$ $\mathrm{dL}$ (normocytic), leukocytosis $13.6 \mathrm{k} / \mathrm{ul}$ with left shift, urinalysis with $1+$ protein, $3+$ blood, 30-50 red blood cells, and urine total protein of $682 \mathrm{mg} / 24 \mathrm{~h}$ with serum creatinine of $0.5 \mathrm{mg} / \mathrm{dL}(0.44$ $1.00 \mathrm{mg} / \mathrm{dL}$ ). Due to the presence of bloody airway secretions from Endotracheal Tube (ET), there was increased concern for pulmonary-renal syndrome, diffuse alveolar hemorrhage and an underlying vasculitis. Intravenous methylprednisolone was initiated at $250 \mathrm{mg}$ every 12 hours and rheumatology service was requested for concern of vasculitis who further increase the methylprednisolone to pulse dose of $250 \mathrm{mg}$ every 6 hours. FiO2 requirement of patient on mechanical ventilation started decreasing after initiating steroids. Additional labs revealed negative Anti Nuclear Antibody titer (ANA), highly positive perinuclear Anti-Neutrophil Cytoplasmic Antibody (p-ANCA) at titer 1:5120 $(<1: 20)$, positive anti-myeloperoxidase antibody MPO at 265 (0-19 AU/mL), negative Serine Protease 3 (PR$3)$ at 1 (0-40 AU/ml). Anti-glomerular basement membrane antibody, anti-double stranded DNA (ds DNA), anti-smith, anti Ribonucleoprotein antibodies (RNP), Sjogren Syndrome A antibody (SSA), Sjogren Syndrome B antibody (SSB), rheumatoid factor, cryoglobulin were all negative. Complements (C3, C4) and Angiotensin Converting Enzyme (ACE) level were normal. Open lung biopsy was performed on 3rd day of intubation. The final pathology report (Figure 3 biopsy photomicrographs) revealed mainly diffuse alveolar damage with hyaline membrane formation, foci of leukocytoclastic vasculitis/capillaritis, focal organizing pneumonia and interstitial fibrosis consistent with microscopic polyangiitis. 
The patient received pulse dose of Methylprednisolone for 5 days, followed by Prednisone $1 \mathrm{mg} / \mathrm{kg}$. Her respiratory status started improving with decreased oxygen requirement (FiO2 $50 \%$ from $\mathrm{FiO} 2$ of $80 \%$ ) on Assist Controlled mode ventilation and patient became alert on mechanical ventilation when sedation was decreased. Antibiotics were stopped, CRP trended down to 1.86 (0-0.744 $\mathrm{mg} / \mathrm{dl})$ and blood tinged secretions from endotracheal tube decreased Rituximab therapy was discussed with family for MPA treatment. Family wanted their patient to be transferred to tertiary facility for further investigation. The patient was transferred to specialized facility where she received Rituximab induction therapy.

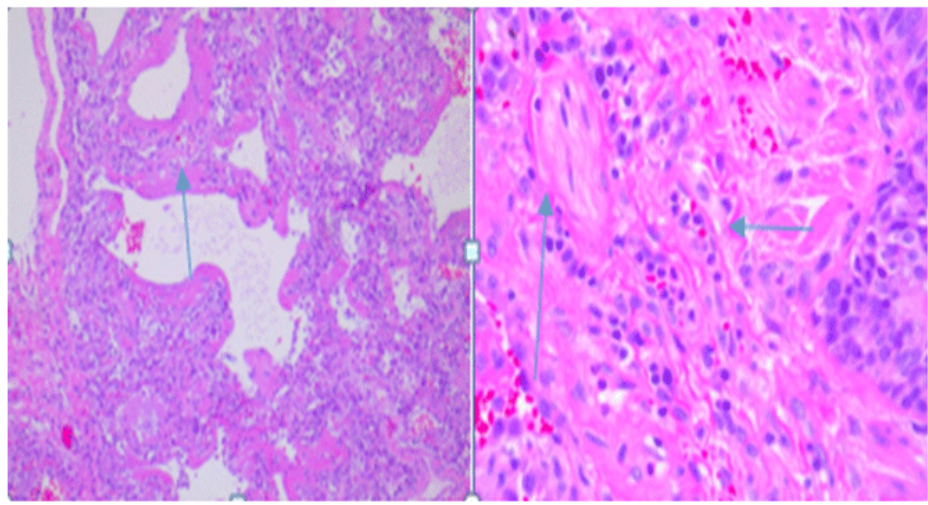

Figure 3: Representative photomicrographs of pulmonary changes consistent with microscopic polyangiitis. A. Diffuse alveolar damage with alveolar and interstitial edema and hyaline membranes lining alveolar surface (H\&E, x100). B. Capillaries with neutrophilic infiltrate (capillaritis) (H\&E, $\mathrm{x} 400)$.

\section{Discussion}

Microscopic PolyAngiitis (MPA) is an uncommon multisystem disease, characterized by inflammatory, destructive vasculitis, primarily of the small blood vessels, leading to tissue ischemia, necrosis and in some cases, hemorrhage [8, 14 \& 15]. Patients often present with nonspecific signs of systemic illness as well as organ based symptoms [16]. Skin, kidney, peripheral nerves and lungs are usual sites involved [16]. We are reporting a case of hyaline membranes formation on histopathology of patient with microscopic polyangiitis. Often patients have perinuclear Anti-Neutrophil Cytoplasmic Antibodies (ANCAs), which primary target Myeloperoxidase (Mpo), protein found in neutrophils granules. However, not all MPA patients appear to have p-ANCAs [17]. Biopsy of affected tissue is usually critical to establishing a definitive diagnosis [18].

MPA pathogenesis is thought to involve an initiating event in genetically susceptible individuals that triggers activation of Antigen-Presenting Cells (APCs) and release of proinflammatory cytokines, subsequent activation and differentiation of T-cells, B-cells and formation of Anti-Neutrophil Cytoplasmic Antibodies (ANCAs), priming neutrophils to interact with ANCAs with subsequent adherence to vessels walls and damage to the endothelium/perivascular tissues [19-20]. This inflammation leads to vessels occlusion and/or to thinning of vessel walls, perivascular hemorrhage and extravasation of macrophages and neutrophils, fibrinoid necrosis, which results in myeloperoxidase induced damage of involved organ leading to glomerulonephritis, skin rashes, myalgia, respiratory failure and other presentations [20, 21-25]. Some cases without ANCA association have also been reported so more studies are required to further elaborate the pathophysiology of MPA $[9,26]$.
Pulmonary involvement in MPA is common and has been studied to explain the variable presentation of MPA that may manifest as unresolving pneumonia, diffuse panbronchiolitis, pulmonary aneurysm, interstitial lung disease, pulmonary nodules and cavitary lesion [18, 21, 22, 27, 28 \& 33]. Specific indirect evidence of vasculitis from imaging studies (x-ray, CT, angiography, etc.) should be carefully reviewed [18]. None of them is diagnostic of MPA but can be suggestive of diagnosis when combined with other findings of renal, skin, musculoskeletal system or peripheral nerve involvement and positive serological markers.

Tissue biopsy is the backbone for MPA diagnosis and is of variable nature. During literature review we found various clinical presentations with spectrum of biopsy findings concerning for MPA. Small-artery vasculitis with considerable fibrin deposition and pauci-immune necrotizing inflammation of the artery wall (arterioles, capillaries and venules), vessel lumen occlusion, without presence of granuloma and eosinophilic infiltrates [10, 11 \& 29]. But in some, the change is limited to infiltration with neutrophils, which become fragmented as they follow the vessel wall (leukocytoclasia) [25]. The term leukocytoclastic angiitis is given to such lesions, most commonly found in post capillary venules. This vascular damage may present as pauci-immune glomerulonephritis intestinal bleeding organ infarctions cutaneous leukocytoclastic vasculitis pulmonary aneurysm and cerebral aneurysm with spectrum of other clinical presentations $[10,11,14,15,25,28 \& 29]$.

Pulmonary capillaritis and necrotizing inflammation without presence of granuloma is confirmatory for MPA diagnosis in the presence of other clinically suggestive findings [3, 4, 21 \& 22]. Pulmonary Fibrosis (PF) is an uncommon manifestation 
observed in patients with ANCA-Associated Vasculitis (AAV), particularly microscopic polyangiitis. These patients have been described only in case reports or small retrospective case series [24, 30-32]. Patients with pulmonary fibrosis associated with AAV seem to have a worse prognosis [31]. Alveolar hemorrhage is another finding that has been reported in previous case reports that has increased mortality if go unrecognized with MPA as etiology $[4,8]$. An acute alveolar hyaline membrane formation, which corresponds to Diffuse Alveolar Damage (DAD) and clinical condition of Acute Respiratory Distress Syndrome (ARDS), is a common pathway of severe alveolar damage secondary to many causes, including shock, infection, inhalation injury, medication, metabolic disorders, hematologic disorder (including disseminated intravascular coagulation), and many others [12, 13 \& 32]. In present case, acute alveolar hyaline membrane formation was discovered to be the result of microscopic polyangiitis that opens new horizon for further studies to consider this presentation suggestive of MPA in the presence of supportive clinical findings.

Preliminary diagnosis of ANCA-associated vasculitis should be made in order to start treatment and biopsy should be obtained as soon as possible to differentiate MPA from infection, malignancy and other vasculitis mimickers in presence of ANCA positivity [1]. Once diagnosis is established, treatment has to be initiated with systemic glucocorticoids and immunosuppressive therapy that includes rituximab or cyclophosphamide induction therapy, as well as plasmapheresis in some cases [34-36].

\section{Conclusion}

Microscopic polyangiitis can be a challenging diagnosis to make and can be life threatening if not properly diagnosed and treated in a timely manner. Pulmonary manifestations range from antecedent interstitial fibrosis to alveolar hemorrhage secondary to diffuse alveolar damage and pulmonary capillaritis. In present case, prominent acute alveolar hyaline membrane formation was discovered to be the result of microscopic polyangiitis. Hyaline membranes have never been reported in relation to MPA. Further studies are needed to reinforce alveolar hyaline membrane formation to be recognized as part of rare biopsy findings of MPA.

\section{Competing interests}

The authors declare that there is no conflict of interests regarding the publication of this paper.

\section{References}

1. Miloslavsky EM, Stone JH and Unizony SH. Challenging mimickers of primary systemic vasculitis. Rheum Dis Clin North Am. 2015;41(1):141-160. Doi: 10.1016/j.rdc.2014.09.011.

2. Bosch X. Microscopic polyangiitis (microscopic polyarteritis) with late emergence of generalised Wegener's granulomatosis. Ann Rheum Dis. 1999;58(10):644-647.

3. Wilke L, Prince-Fiocco M and Fiocco GP. Microscopic polyangiitis: a large single-center series. J clin Rheumatol. 2014;20(4):179-182. Doi: 10.1097/RHU.0000000000000108.
4. Fatma LB, El Ati Z, Lamia R, Aich DB, Madiha K and Wided S. et al. Alveolar hemorrhage and kidney disease: characteristics and therapy. Saudi J Kidney Dis Transpl. 2013;24(4):743-750.

5. Wang CC, Shiang JC, Tsai MK, Chen IH, Lin SH and Chu P. et al. Prompt plasmapheresis successfully rescue pulmonary-renal syndrome caused by ANCA-negative microscopic polyangiitis. Clin Rheumatol. 2009;28(12):1457-1460. Doi: 10.1007/s10067-009-1264-2.

6. Phatak S, Aggarwal A, Agarwal V, Lawrence A and Misra R. Antineutrophil cytoplasmic antibody (ANCA) testing: Audit from a clinical immunology laboratory. Int J Rheum Dis. 2017;20(6):774778. Doi: 10.1111/1756-185X.12926.

7. Digby JW, Tinwell B, Sheldon J and Wang J. The Missing Antibody: The Pitfalls of ANCA Testing. Am J Med. 2017;130(3):e93-e96. Doi: 10.1016/j.amjmed.2016.11.003.

8. Lauque D, Cadranel J, Lazor R, Pourrat J, Ronco P and Guillevin L. et al. Microscopic polyangiitis with alveolar hemorrhage. A study of 29 cases and review of the literature. Groupe d'Etudes et de Recherche sur les Maladies "Orphelines" Pulmonaires (GERM"O"P). Medicine (Baltimore). 2000;79(4):222-233.

9. Sharon A, Chung and Philip Seo. Microscopic Polyangiitis. Rheum Dis Clin North Am. 2010;36(3):545-558. Doi: 10.1016/j.rdc.2010.04.003.

10. Rutgers A, Sanders JS and Kallenberg CG. Pauci-Immune Necrotizing Glomerulonephritis. Rheum Dis Clin North Am. 2010;36(3):559-572. Doi: 10.1016/j.rdc.2010.05.002.

11. Fogo AB, Lusco MA, Najafian B and Alpers CE. AJKD Atlas of Renal Pathology: Pauci-immune Necrotizing Crescentic Glomerulonephritis. Am J Kidney Dis. 2016;68(5):e31-e32. Doi: 10.1053/j.ajkd.2016.09.002.

12. Cardinal-Fernández P, Lorente JA, Ballén-Barragán A and MatuteBello G. Acute Respiratory Distress Syndrome and Diffuse Alveolar Damage. New Insights on a Complex Relationship. Ann Am Thorac Soc. 2017;14(6):844-850. Doi: 10.1513/AnnalsATS.201609-728PS.

13. Younsuck Koh. Update in acute respiratory distress syndrome. J Intensive Care. 2014;2(1). Doi: 10.1186/2052-0492-2-2.

14. Ueda S, Matsumoto M, Ahn T, Adachi S, Oku K and Takagi M. Microscopic polyangiitis complicated with massive intestinal bleeding. J Gastroenterol. 2001;36(4):264-270.

15. Ku BD and Shin HY. Multiple bilateral non-hemorrhagic cerebral infarctions associated with microscopic polyangiitis. Clin Neurol Neurosurg. 2009;111(10):904-906.

16. Guillevin L, Durand-Gasselin B, Cevallos R, Gayraud M, Lhote F, Callard $\mathrm{P}$ and Amouroux J. Microscopic polyangiitis: clinical and laboratory findings in eighty-five patients. Arthritis Rheum. 1999;42(3):421-430. Doi: 10.1002/1529-0131(199904)42:3<421::AID-ANR5>3.0.C0;2-6.

17. Gómez-Puerta JA, Hernández-Rodríguez J, López-Soto A and Bosch $\mathrm{X}$. Antineutrophil cytoplasmic antibody-associated vasculitides and respiratory disease. Chest. 2009;136(4):1101-1111. Doi: 10.1378/ chest.08-3043.

18. Pesci A and Manganelli P. Respiratory system involvement in antineutrophil cytoplasmic-associated systemic vasculitides: clinical, 
pathological, radiological and therapeutic considerations. Clin Pract. 2014 Jul 4; 4(2):653.

19. Kallenberg CG, Heeringa P and Stegeman CA. Mechanisms of Disease: pathogenesis and treatment of ANCA-associated vasculitides. Nat Clin Pract Rheumatol. 2006;2(12):661-670. Doi:10.1038/ncprheum0355

20. Guilpain P, Servettaz A, Goulvestre C, Barrieu S, Borderie D and Chéreau C. et al. Pathogenic effects of antimyeloperoxidase antibodies in patients with microscopic polyangiitis. Arthritis Rheum. 2007;56(7):2455-2463. Doi:10.1002/art.22741.

21. Ter Maaten JC, Franssen CF, Gans RO, Van Schijndel RA and Hoorntje SJ. Respiratory Failure in ANCA-Associated Vasculitis. Chest 1996;110(2):357-362.

22. Sourla E, Bagalas V, Tsioulis H, Paspala A, Akritidou S and Pataka A. et al. Acute respiratory failure as primary manifestation of antineutrophil cytoplasmic antibodies-associated vasculitis. Clin Pract. 2014;4(2):653. Doi: 10.4081/cp.2014.653.

23. Schirmer JH, Wright MN, Vonthein R, Herrmann K, Nölle B and Both M. et al. Clinical presentation and long-term outcome of 144 patients with microscopic polyangiitis in a monocentric German cohort. Rheumatology (Oxford). 2016;55(1):71-79. Doi: 10.1093/ rheumatology/kev286.

24. Birnbaum J, Danoff S, Askin FB and Stone JH. Microscopic polyangiitis presenting as a "pulmonary-muscle" syndrome: is subclinical alveolar hemorrhage the mechanism of pulmonary fibrosis? Arthritis Rheum. 2007;56(6):2065-2071. Doi:10.1002/art.22633.

25. Kawakami T, Soma Y, Saito C, Ogawa H, Nagahuchi Y and Okazaki T. et al. Cutaneous manifestations in patients with microscopic polyangiitis: two case reports and a minireview. Acta Derm Venereol. 2006;86(2):144-147. Doi:10.2340/00015555-0034.

26. Wang R, Yang XC, Zhou SJ and Sun GY. Anti-neutrophil cytoplasmic antibody-negative microscopic polyangiitis: A case report and literature review. Exp Ther Med. 2015;10(2):749-752. Doi:10.3892/ etm.2015.2523.

27. Park J, Banno S, Sugiura Y, Yoshikawa K, Naniwa T and Wakita K. et al. Microscopic polyangiitis associated with diffuse panbronchiolitis. Intern Med. 2004;43(4):331-335.
28. Ortiz-Santamaria V, Olivé A, Holgado S and Muchart J. Pulmonary aneurysms in microscopic polyangiitis. Clin Rheumatol. 2003;22(6):498-499. Doi:10.1007/s10067-003-0765.

29. Takahashi T, Katayama W, Kujiraoka Y, Yamamoto $T$ and Matsumura A. A Case of a Ruptured Cerebral Aneurysm Associated with Microscopic Polyangiitis. No Shinkei Geka. 2018;46(2):117-122. Doi: 10.11477/ mf.1436203686.

30. Katsumata Y, Kawaguchi $Y$ and Yamanaka H. Interstitial Lung Disease with ANCA-associated Vasculitis. Clin Med Insights Circ Respir Pulm Med. 2015;9(Suppl 1):51-56. Doi:10.4137/CCRPM.S23314.

31. Comarmond C, Crestani B, Tazi A, Hervier B, Adam-Marchand S and Nunes H. et al. Pulmonary Fibrosis in Antineutrophil Cytoplasmic Antibodies (ANCA)-Associated Vasculitis: A Series of 49 Patients and Review of the Literature. Medicine (Baltimore) 2014;93(24):340349. Doi: 10.1097/MD.0000000000000217.

32. Tzelepis GE, Kokosi M, Tzioufas A, Toya SP, Boki KA and Zormpala A. et al. Prevalence and outcome of pulmonary fibrosis in microscopic polyangiitis. Eur Respir J. 2010;36(1):116-121. Doi: 10.1183/09031936.00110109. Halide Nur Urer, Gokhan Ersoy and Emine Dilek Yllmazbayhan. Diffuse Alveolar Damage of the Lungs in Forensic Autopsies: Assessment of Histopathological Stages and Causes of Death. Scientific World Journal. 2012;2012:657316. Doi: 10.1100/2012/657316.

33. Chung MP, Yi CA, Lee HY, Han J and Lee KS. Imaging of Pulmonary Vasculitis. Radiology. 2010;255(2):322-341. Doi: 10.1148/ radiol.10090105.

34. Stone JH, Merkel PA, Spiera R, Seo P, Langford CA and Hoffman GS. et al. Rituximab versus cyclophosphamide for ANCA-associated vasculitis. N Engl J Med. 2010;363(3):221-232. Doi:10.1056/NEJMoa0909905.

35. Rabrenovic V, Kovacevic Z, Jovanovic D, Rabrenovic M, Dimitrijevic J and Skataric V. et al. Microscopic polyangiitis. Vojnosanit Pregl. 2001;58(3):323-328.

36. Guillevin L. Treatment of ANCA associated systemic necrotizing vasculitides. Bull Acad Natl Med. 2008;192(6):1157-1187. 